\title{
SCAMPER-3-5 para generación de ideas y su análisis de valor.
}

\section{MME Alemanya ${ }^{a}$ Llanos Cuenca ${ }^{a}$, Leonor Ruiz ${ }^{\text {a }}$ Andrés Boza ${ }^{a}$}

${ }^{a}$ Departamento de Organización de Empresas. Universitat Politècnica de València. Equipo de Innovación Educativa AEGECATE. (mareva@omp.upv.es, llcuenca@omp.upv.es, 1ruiz@omp.upv.es, aboza@omp.upv).

\begin{abstract}
At the Universitat Politècnica de València, thirteen competency dimensions have been defined, among which is the competency of creativity, innovation and entrepreneurship. This competency has become in recent years one of the most demanded in the labor market. This paper presents the design and development of the SCAMPER-3-5 activity designed to foster the acquisition of the transversal competency. The proposed technique is based on the combination of the SCAMPER and 6-3-5 extended techniques, to eliminate the limitations encountered and to design an activity that reaches the whole process associated to creativity, innovation and entrepreneurship. This process includes the phases of search for opportunities, generation of ideas, action plan and value analysis. The proposed activity is easily applicable and transferable to any subject, and can be used in any context. It contributes to the development of transversal competence in students.
\end{abstract}

Keywords: Transversal competency, Creativity, Innovation, Entrepreneurship, SCAMPER, 6-3-5

\section{Resumen}

En la Universitat Politècnica de València se han definido un total de trece dimensiones competenciales, entre las que se encuentra, la competencia de creatividad, innovación y emprendimiento. Esta competencia, se ha convertido en los últimos años en una de las más demandadas en el mercado laboral. En este trabajo se presenta el diseño y desarrollo de la actividad SCAMPER-3-5 diseñada para favorecer la adquisición de la competencia transversal. La técnica propuesta se basa en la combinación de las técnicas SCAMPER y 6-3-5 extendida, para eliminar las limitaciones encontradas y diseñar una actividad que alcance todo el proceso asociado a la competencia de creatividad, innovación y emprendimiento. Dicho proceso incluye las fases de búsqueda de oportunidades, generación de ideas, plan de acción y análisis de valor. La actividad propuesta es fácilmente aplicable y transferible a cualquier asignatura, pudiendo ser utilizada en cualquier contexto. Contribuye por tanto al desarrollo de la competencia transversal en los alumnos.

Palabras clave: Competencia transversal, Creatividad, Innovación, Emprendimiento, SCAMPER, 6-3-5. 


\section{Introducción}

La innovación se ha convertido en un requisito para la ventaja competitiva de las organizaciones, por lo que es en un aspecto cada vez más importante y demandado. Favorecer la adquisición de la competencia en innovación es, por tanto, uno de los aspectos a resolver (Boza \& Cuenca 2014), especialmente en las enseñanzas universitarias que preparan al egresado para su inserción en el mundo laboral.

En la UPV Se han definido un total de 13 dimensiones competenciales. DC1-Comprensión e integración; DC2-Aplicación del pensamiento práctico; DC3-Análisis y resolución de problemas; DC4-Innovación, creatividad y emprendimiento; DC5-Diseño y proyecto; DC6Trabajo en equipo y liderazgo; DC7-Responsabilidad ética y profesional; DC8Comunicación efectiva; DC9-Pensamiento crítico; DC10-Conocimiento de los problemas contemporáneos; DC11-Aprendizaje permanente; DC12-Planificación y gestión del tiempo; DC13-Instrumental específica.

La adquisición de las anteriores competencias se establece en base al nivel de alcance de unos determinados resultados de aprendizaje en los que se desglosan estas competencias.

La manera que en que transmiten los conocimientos para la consecución de los resultados de aprendizaje puede ser a través de las actividades desarrolladas y objetos de aprendizaje complementarios(Fernández-Diego et al. 2015) que permitan su evaluación (Cuenca, Alarcón, Boza, et al. 2016). Diversas actividades han sido propuestas y aplicadas por los autores, para facilitar la adquisición de la competencia de creatividad, innovación y emprendimiento en las aulas (Ruiz et al. 2015); (Boza et al., 2016).

La Universitat Politècnica de València recoge las directrices para la incorporación de las competencias transversales en las titulaciones. El sistema de evaluación debe procurar una evaluación continua del alcance de los resultados de aprendizaje y de las competencias previstas en la asignatura o la materia objeto de evaluación. Es un objetivo transformar el proceso de enseñanza-aprendizaje para hacer del alumno su principal protagonista $\mathrm{y}$, en este sentido, es necesario orientar las programaciones y metodologías docentes de las asignaturas centrándolas en el aprendizaje de los estudiantes y no exclusivamente en las horas lectivas. La clave está en incluir en el programa de cada asignatura un conjunto de actividades que aseguren el aprendizaje del alumno e introducir los mecanismos necesarios para motivar al alumno a realizarlas.

Para garantizar la adquisición de la competencia en las titulaciones, se han definido asignaturas punto de control que garanticen que la competencia se trabaja y se evalúa en el aula. Sin embargo el número de asignaturas candidatas a ser punto de control, para esta competencia es inferior al que se puede encontrar para otras competencias transversales, el profesorado encuentra dificultades para incorporarla en sus asignaturas. Es por esto, que la competencia transversal, creatividad, innovación y emprendimiento, se presenta como un reto a la hora de definir actividades que favorezcan su adquisición y transferencia a distintas asignaturas y puedan ser utilizadas en diversos contextos.

(c) EY-NC-ND 2017, Universitat Politècnica de València 


\section{Objetivos}

El objetivo del trabajo es presentar el diseño y desarrollo de la actividad SCAMPER-3-5 para la adquisición de la competencia transversal creatividad, innovación y emprendimiento.

Se puede entender esta competencia como un proceso que parte de la búsqueda de oportunidades, pasando por la generación de ideas, para finalmente, llevar a cabo su implementación a través de un plan de acción y su análisis de valor (Figura 1).

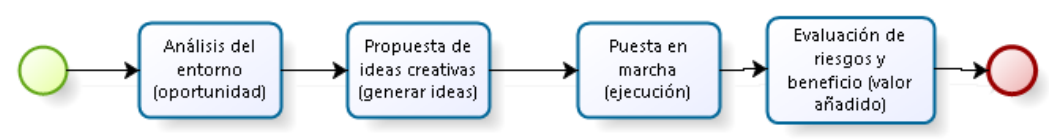

Figura 1 Proceso asociado a la competencia transversal creatividad, innovación y emprendimiento

La actividad desarrollada aborda todas las fases del proceso.

\section{Desarrollo de la Innovación}

En primer lugar se revisaron y aplicaron las técnicas básicas para generación de ideas que se tomaron como base para el diseño de la innovación y posteriormente se desarrolló la innovación propuesta.

\subsection{Técnicas básicas: Scamper y 6-3-5}

\subsubsection{Técnica SCAMPER}

Scamper es una técnica de creatividad o de desarrollo de ideas creativas elaborada por Bob Eberle a mediados del siglo 20 (Eberle 2008). Esta técnica permite la generación de nuevas ideas al realizar acciones sobre una idea base. Parte de la base de que un producto, servicio, o proceso existentes, tanto si son propios como si son de la competencia, se pueden mejorar, si aplico una serie de verbos y preguntas relacionadas y persigo las respuestas para ver donde le llevan.

Básicamente es una lista de chequeo que corresponde a las letras de la técnica y puede incluir las siguientes preguntas:

- S: Sustituir. ¿Quién más puede ser sustituido?¿Qué más puede ser sustituido? ¿Podemos cambiar las reglas?¿Otros ingredientes?¿Otros materiales?

- C: Combinar. ¿Qué ideas se pueden combinar?¿Podemos combinar propósitos, intenciones? ¿Qué tal una colección?¿Qué tal una mezcla, una aleación, una agrupación? ¿Combinar unidades?

- A: Adaptar. ¿Qué más es como esto?¿Qué otra idea te sugiere esto?¿El pasado nos ofrece algún paralelismo?¿Qué podríamos copiar?¿Qué idea podríamos incorporar?

- M: Magnificar. ¿Qué podemos magnificar, ampliar, o extender?¿Qué podemos exagerar?¿Qué podemos sobredimensionar?¿Qué podemos añadir?¿Más tiempo? 
¿Más fuerte? Modificar ¿Cómo lo podemos alterar para mejorarlo? ¿Se puede modificar?¿Hay alguna peculiaridad?¿Cambiar el significado, el color, el movimiento, el sonido, el olor, la forma, la medida? ¿Cambiarle el nombre?

- P: Poner en otros usos. ¿Para qué más se podría usar? ¿Hay nuevas maneras de usarlo tal y como es, tal y como está? ¿Otros usos si lo modificamos?

- E: Eliminar. ¿Qué pasaría sí fuese más pequeño?¿Qué habría que omitir?¿Lo tendría que dividir? ¿Trocearlo? ¿Separarlo en diferentes partes? ¿Reducir? ¿Hacer más eficiente? ¿Hacerlo en miniatura?

- R: Reorganizar. ¿Qué otras organizaciones podrían ser mejores? ¿Intercambiar componentes?¿Un modelo diferente?¿Una distribución diferente?¿Otra secuencia?

Se basa en la idea de que mucho de lo que es nuevo, en realidad es una modificación de lo que ya existe

\section{Limitaciones}

Al aplicar la técnica en grupo, en ocasiones las ideas no surgen con facilidad. Al tener delante la hoja con las 7 letras, el alumno no sabe por cual debe empezar, cambia de opinión a mitad de desarrollo y le resulta difícil saber en qué letra encaja su idea. Por otra parte no existe tiempo limitado por lo que se debe dimensionar la actividad en el aula y marcar los tiempos a criterio del profesor.

\subsubsection{Técnica 6-3-5}

La técnica 6-3-5 es una forma específica de brainwriting (Rohrbach, 1969). Trata de resolverlos problemas típicos en el brainstorming, como son las barreras a la creatividad. 63-5 se refiere a seis personas que desarrollan y anotan tres ideas dentro de un marco de tiempo definido de cinco minutos antes de pasar sus ideas para el compañero. En el siguiente paso cada miembro del equipo tiene que evolucionar ideas, basadas en las dadas a través de su predecesor. Esto puede ocurrir mediante la modificación de las ideas existentes o nuevas ideas inspiradas en los otros datos. Este procedimiento se continúa hasta que cada miembro del equipo recibe su hoja inicial de ideas, lo cual deberá hacerse después de cinco rondas considerando seis participantes. Este paso es desarrollado por grupos a lo largo de 30 minutos. La actividad está específicamente orientada a mejorar la creatividad y la generación de ideas.

\section{Limitaciones}

Cada alumno debe generar un número de ideas relacionadas con el tema indicado. Aunque la técnica es de muy fácil aplicación y delimitada en el tiempo, lo que favorece el desarrollo en el aula, en ocasiones genera frustraciones entre los alumnos. La generación de nuevas ideas puede verse favorecida al leer las ideas de los compañeros, y la retroalimentación que se produce. Sin embargo, la libertad en la generación de ideas, y el límite de tiempo, lleva a no concretar qué se pretende hacer, y a no saber cuál es el objetivo de la idea propuesta, o cómo se llevará a cabo. Por otra parte, si una de las ideas propuestas no está bien planteada y el resto se basa en ella, provoca un efecto en cadena negativo, que obligará a descartar muchas de las ideas que se hayan generado.

(cc) BY-NC-ND 2017, Universitat Politècnica de València 


\subsubsection{Técnica 6-3-5 extendida}

La técnica 6-3-5 extendida es una variación de la anterior, que ha sido propuesta por los autores (Cuenca, Alarcón, Alemany, et al. 2016), considerando que para la competencia de creatividad, innovación y emprendimiento, no solo se necesita generar ideas, sino que éstas aporten valor y sean llevadas a cabo con una actitud emprendedora.

A las etapas de generación de ideas se añaden las fases de Selección (individual y en grupo), Plan de Acción, Análisis de Valor y Debate (Boza et al., 2016). En esta actividad no solo se ha prestado atención a la generación de ideas, sino también al planteamiento propuesto para su puesta en marcha y su análisis de valor. Completando así la competencia transversal CT-4 creatividad, innovación y emprendimiento.

Las limitaciones para la fase de generación de ideas son similares a las que se producen en la técnica básica 6-3-5 para las primeras fases.

\subsection{PropuestaSCAMPER-3-5}

La técnica propuesta se basa en la combinación de las técnicas SCAMPER y 6-3-5 extendida, para por una parte, eliminar las limitaciones encontradas y por otra, diseñar una actividad que alcance todo el proceso asociado a la competencia de creatividad, innovación y emprendimiento.

\section{Fase 1. Identificación de la oportunidad y generación de Ideas}

Al principio de la actividad, cada alumno del grupo dispone de una hoja que se corresponde con cada una de las letras que componen el acrónimo SCAMPER y una tabla con 3 columnas (ideas) y 7 filas (miembros del grupo). Por ejemplo, la primera persona tendrá una hoja con la letra "S" que indica que tiene que colocar en la primera fila de la tabla correspondiente a su hoja, tres ideas encaminadas a "Sustituir" alguno de los elementos de un producto/servicio/elemento/sistema (Figura 2). La segunda persona una hoja con la letra "C" de "combinar" y la misma tabla ya sí con las 7 letras de SCAMPER para las 7 personas del grupo

\begin{tabular}{|c|c|c|c|}
\hline & & S' UMERO: & \\
\hline & 5 para $\mathrm{Di}$ & roductive & Simulación \\
\hline & & ITUIR & \\
\hline $\begin{array}{l}\text { COMPONENEE } \\
\text { GUVPOO }\end{array}$ & $\begin{array}{c}\text { IDEA1 } \\
\text { S-SUSTITIIR }\end{array}$ & $\begin{array}{c}\text { IDEA2 } 2 \\
\text { s-SUSTITIRR }\end{array}$ & $\begin{array}{c}\text { IDEA } 3 \\
\text { S-SUSTITIRR }\end{array}$ \\
\hline 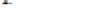 & & & \\
\hline 2 & & & \\
\hline & & & \\
\hline & & & \\
\hline 4. & & & \\
\hline 5. & & & \\
\hline 6 & & & \\
\hline & & & \\
\hline & & & \\
\hline
\end{tabular}

Figura 2 Ejemplo plantilla actividad SCAMPER-3-5 
Los alumnos disponen de 5 minutos para rellenar las 3 casillas correspondientes a las 3 ideas de la letra SCAMPER que corresponda a su hoja. Finalizados los 5 minutos, los alumnos se intercambiarán las hojas en el sentido de las agujas del reloj y tendrán de nuevo otros 5 minutos para aportar 3 ideas pero ahora para la letra de SCAMPER que aparezca en la nueva hoja. Tras 7 iteraciones (35minutos) todos los alumnos del grupo deben haber rellenado una fila de cada una de las 7 hojas con 3 ideas.

\section{Fase 2. Selección de ideas individual}

Selección de ideas individual para cada una de las letras SCAMPER (5- 10min). En esta fase, cada uno de los alumnos se queda con la última hoja que ha rellenado que se corresponderá con una letra de SCAMPER y en la que se habrán generado 21 ideas. Como existen 7 hojas, un por cada letra de la palabra SCAMPER, en total se habrán generado $21 * 7$ ideas. Cada alumno tendrá que escoger 5 ideas de entre las 21 existentes una hoja para la letra de SCAMPER de que disponga en ese momento según los siguientes criterios:

- Idea más original

- La que implica menor riesgo

- La más urgente

- La que aporta más benefício

- La que aporta más valor

El análisis de riesgo, urgencia, beneficio y valor, variará en función del contexto donde se lleve a cabo la innovación. La originalidad puede medirse en función de su novedad, su capacidad de comunicar el objetivo perseguido y lo atractivo de la propuesta.

\section{Fase 3. Selección de ideas grupal.}

Selección de ideas grupal (10-15min) finalizado el proceso anterior, se ponen en común las 5 ideas seleccionadas por cada participante. De nuevo se seleccionan 5 de acuerdo a los criterios anteriores.

\section{Fase4. Plan de acción}

Por último en base a los criterios definidos en el grupo, se selecciona una de las ideas a implantar (10 minutos) (la más urgente, la que mayor beneficio aporta, ...) o bien se genera una idea integrada. El grupo propone un plan de acción inicial para implantar la idea seleccionada.

\section{Fase5. Análisis de valor}

El grupo define (10 minutos) en base a qué parámetros se espera medir el valor de la/s nueva/s alternativa/s de manera que se pueda justificar su utilidad.

\section{Fase6. Presentación de la actividad por el representante del grupo}

El grupo elige un representante que deberá exponer ante sus compañeros:

- Alternativa seleccionada

- Valor esperado: para el cliente, empresa o sociedad

- Indicadores definidos para medir el valor de la alternativa generada.

(cc) EY-NC-ND 2017, Universitat Politècnica de València 


\section{Fase7. Coevaluación}

La coevaluación (5min) es la fase final de la actividad, que junto con la evaluación del profesor conforman la puntuación final de la actividad. Es recomendable que la coevaluación se realice por medio de una rúbrica aunque puede hacerse sin ella de manera que los alumnos ordenan de mejor a peor alternativa las ideas planteadas salvo las de su grupo y se le otorga una puntuación predefinida dependiendo de la posición que ocupa sobre el total del número de grupos: la máxima puntuación se le asignará a la idea situada en el primer puesto.

\section{Fase 8. Selección de la mejor idea}

Finalmente, se suma la puntuación obtenida para cada idea por cada uno de los grupos, seleccionando como mejor idea la que obtenga mayor puntuación.

Con esta técnica se da respuesta a las limitaciones encontradas anteriormente, consiguiendo las siguientes ventajas:

- En cada momento cada alumno sabe hacia dónde debe enfocar su solución, y por tanto su esfuerzo, les hace actuar más rápido.

- Se introduce el factor tiempo en la técnica SCAMPER lo que favorece la aplicación en el aula.

- El alumno no se agota o se enroca en generar ideas en la misma dirección (cambiar nivel, cambiar texto, cambiar persona,...), sino que se le ayuda a tener un punto de vista distinto (sustituir, combinar, adaptar....).

- Los alumnos formalizan mejor su idea al ir enfocada a una pregunta concreta.

- Los alumnos completan el proceso asociado a la competencia de creatividad, innovación y emprendimiento. Desde la identificación de una oportunidad para la generación de ideas, hasta la definición del plan de acción y análisis de valor.

\section{Resultados}

\subsection{Aplicación}

\subsubsection{Contexto}

La actividad SCAMPER-3-5 se aplicó en una de las prácticas de aula de la asignatura "Métodos Cuantitativos de Organización Industrial". Se trata de una asignatura anual de tercer curso del Grado de Ingeniero de Organización de la ETSII con aproximadamente 80 alumnos matriculados. En esta asignatura los alumnos desarrollan modelos y aplican técnicas y herramientas matemáticas y de simulación para la resolución de múltiples problemas relacionados con la Ingeniería de Organización. La mayoría de estos problemas tiene como foco principal el proceso de toma de decisiones en el que se debe seleccionar la/s alternativa/s que optimice/n o alcance/n de manera satisfactoria uno o varios objetivos, al tiempo que respetan las restricciones del sistema con objeto de asegurar su implementación. 
La CT04-Innovación, creatividad y emprendimiento tiene una especial relevancia a la hora generar diversas alternativas, evaluarlas e implementar la alternativa seleccionada. Además los modelos diseñados como ayuda a la toma de decisiones requieren no sólo de ciencia (conocimientos previos) sino también de un cierto arte (creatividad).

\subsubsection{Desarrollo}

En la asignatura anual de tercer curso del Grado de Ingeniero de Organización de la ETSII, Métodos Cuantitativos de Organización Industrial, se aplicó la actividad SCAMPER-3-5.

La actividad propuesta pretendía que los alumnos fueran capaces de plantear alternativas de diseño/funcionamiento de un supermercado, cuyo comportamiento pudiera ser evaluado posteriormente a través de la simulación. En la clase previa, se les proyectó un video sobre la metodología SCAMPER para, en la clase en cuestión, explicarles la actividad completa.

\subsection{Resultados de la innovación}

\subsubsection{Evaluación}

Se formaron 7 grupos y una vez finalizada la actividad, un representante del grupo salió a la pizarra a explicar su idea para mejorar un supermercado bajo el lema "el carrito inteligente". Los alumnos puntuaron a cada grupo, de manera que se eligió como mejor idea la de mayor puntuación. La actitud de los alumnos fue muy participativa y algunas de las ideas generadas fueron muy interesantes.

\subsubsection{Evidencias}

Se muestran a continuación algunas de las evidencias recogidas durante el desarrollo de la actividad.

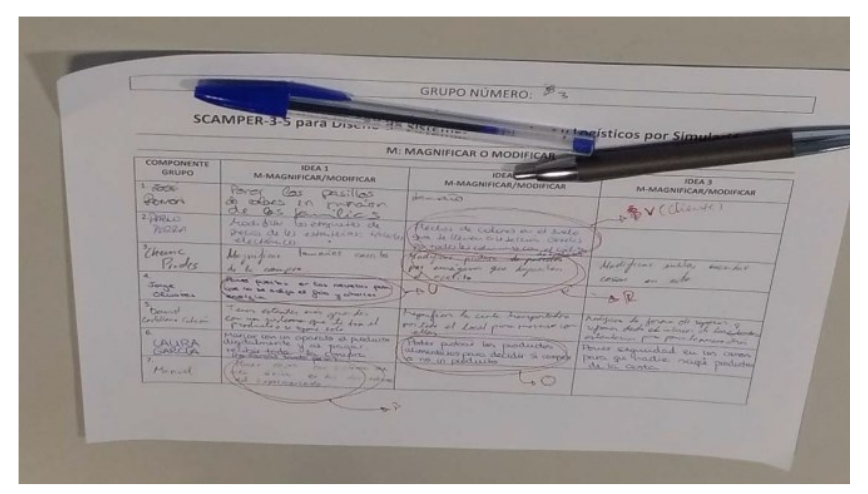

(c) EY-NC-ND 2017, Universitat Politècnica de València 

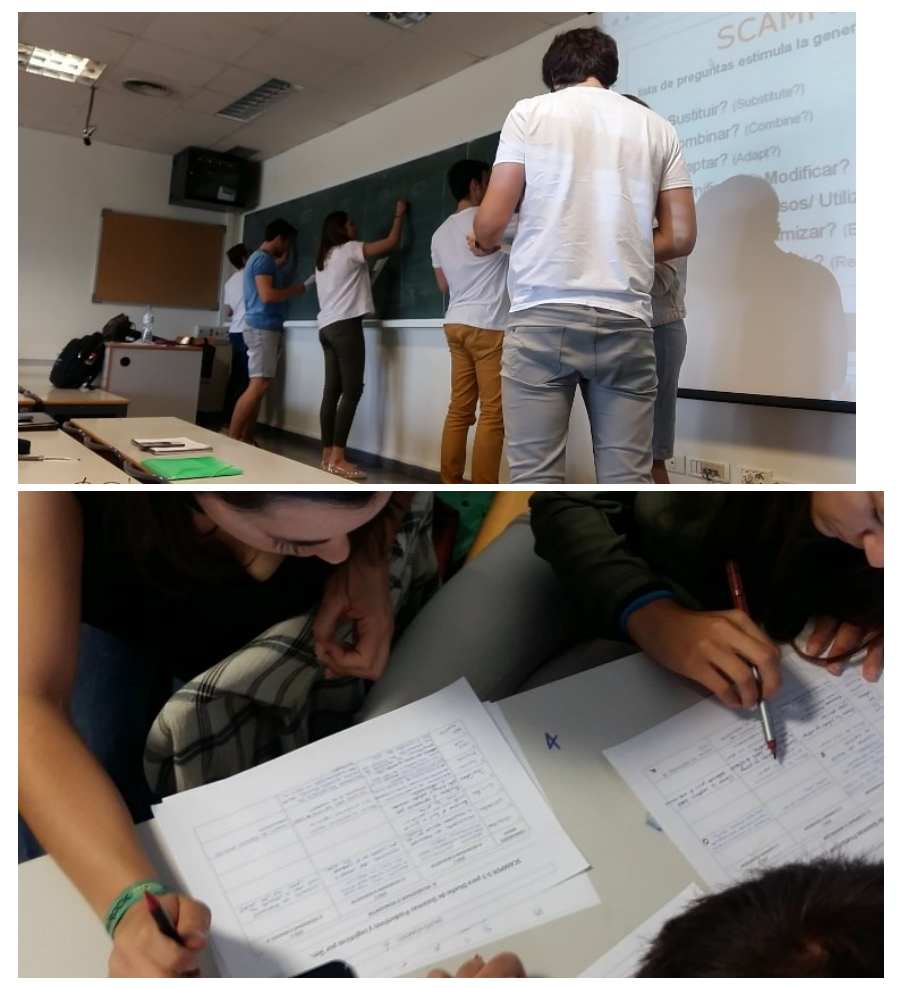

\section{Conclusiones}

La actividad propuesta permite abordar la competencia transversal de creatividad, innovación y emprendimiento de manera completa, pasando por las diferentes fases, identificación de la oportunidad, generación de ideas, plan de acción y análisis de valor. Consigue reducir las limitaciones identificadas en las técnicas básicas que han servido como punto de partida al diseño de la actividad.

La actividad propuesta es fácilmente aplicable y transferible a cualquier asignatura, pudiendo ser utilizada en cualquier contexto. Contribuye por tanto al desarrollo de la competencia transversal en los alumnos. Se ha ampliado el número de actividades que se pueden aplicar en el aula, lo que contribuye también a reducir el temor a esta competencia transversal que algunos profesores habían manifestado, y por tanto podrá aumentar el número de asignaturas punto de control.

La actividad fue valorada muy positivamente, aunque algunos alumnos indicaron la falta de tiempo en algunas fases. Esto puede ser debido a que los alumnos no están familiarizados con este tipo de técnicas.

Recomendamos, que la adquisición de la competencia no se trabaje y evalúe con una sola actividad puntual, sino en varias actividades a lo largo del curso, para ello se puede iniciar con alguna actividad previa y sencilla, sólo con las técnicas básicas, de manera que se vayan familiarizando y poder ser más ágiles en la ejecución de la actividad propuesta. 
Como futura línea de investigación se propone comparar los resultados de la coevaluación con/sin rúbrica, y analizar con más detalle el valor de la actividad propuesta.

\section{Referencias}

Boza, A. \& Cuenca, L., 2014. Strategy of online assessment in case study methodology. A particular case in the subject enterprise computers tools. ICERI2014 Proceedings, pp.32073211.

Boza A., Fernandez-Diego M., Ruiz L., Gordo M.L., Alemany M., Alarcón F., Cuenca L (2016). Transversal Competences as a Medium of Teaching. The case of creativity, innovation and entrepreneurship. 5th International Conference on Strategic Innovative Marketing. September 23-26, 2016 . Athens, Greece

Cuenca, L., Alarcón, F., Alemany, M.M.E., et al., 2016. La técnica 6-3-5 extendida, para la competencia de creatividad, innovación y emprendimiento. https://riunet.upv.es/handle/10251/68332.

Cuenca, L., Alarcón, F., Boza, A., et al., 2016. Rubric to assess the competence of innovation, creativity and enterpreneurship in bachelor degree. Brazilian Journal of Operations \& Production Management, 13(1), pp.118-123.

Eberle, B., 2008. Scamper : creative games and activities for imagination development,

Fernández-Diego, L. et al., 2015. Metadata, repository and methodology in learning objects. EDULEARN15 Proceedings, pp.4755-4761.

Rohrbach, B., (1969) "Kreativ Nach Regeln - Methode 635, Eine Neue Technik Zum Lösen VonProblemen", Absatzwirtschaft, 12, No.19, 1969, pp. 73-75.

Ruiz, L. et al., 2015. Implementación de actividades de aprendizaje y evaluación para el desarrollo de competencias genéricas: un caso práctico de aplicación de técnicas de Pensamiento de Diseño, y evaluación mediante rúbricas, de las competencias de Creatividad, Innovación y E. In Libro de Actas IN-RED 2015 - Congreso Nacional de Innovación Educativa y de Docencia en Red. Editorial Universitat Politècnica de València, pp. 1074-1088. 